\title{
Emotion Recognition from Facial Expression Based on Fiducial Points Detection and using Neural Network
}

\author{
Fatima Zahra Salmam ${ }^{1}$, Abdellah Madani $^{2}$, and Mohamed Kissi ${ }^{3}$ \\ ${ }^{1,2}$ LAROSERI Laboratory, Faculty of Sciences, University of Chouaib Doukkali, El Jadida-Morocco \\ ${ }^{3}$ LIM Laboratory, Faculty of Sciences and Technologies, University Hassan II, Casablanca-Morocco
}

\begin{abstract}
Article Info
Article history:

Received: Jun 5, 2017

Revised: Nov 30, 2017

Accepted: Dec 16, 2017

Keyword:

Facial expression

Feature selection

Neural network

Supervised Descent Method

Best First

ABSTRACT

The importance of emotion recognition lies in the role that emotions play in our everyday lives. Emotions have a strong relationship with our behavior. Thence, automatic emotion recognition, is to equip the machine of this human ability to analyze, and to understand the human emotional state, in order to anticipate his intentions from facial expression. In this paper, a new approach is proposed to enhance accuracy of emotion recognition from facial expression, which is based on input features deducted only from fiducial points. The proposed approach consists firstly on extracting 1176 dynamic features from image sequences that represent the proportions of euclidean distances between facial fiducial points in the first frame, and faicial fiducial points in the last frame. Secondly, a feature selection method is used to select only the most relevant features from them. Finally, the selected features are presented to a Neural Network (NN) classifier to classify facial expression input into emotion. The proposed approach has achieved an emotion recognition accuracy of $99 \%$ on the CK+ database, $84.7 \%$ on the Oulu-CASIA VIS database, and 93.8\% on the JAFFE database.
\end{abstract}

Copyright (C) 2018 Institute of Advanced Engineering and Science. All rights reserved.

\section{Corresponding Author:}

Abdellah Madani

LAROSERI Laboratory, computer science departement

Faculty of Sciences, University of Chouaib Doukkali, El Jadida - Morocco

Email: madaniabdellah@gmail.com

\section{INTRODUCTION}

As emotions play an implicit role in the communication process, and reflect human behavior, automatic emotion recognition is a task of growing interest. To recognize human emotions, a wide range of features can be used such as facial expression[1, 2], body gesture [2,3], or speech [4, 5]. Giving a computer the capability of emotion recognition (ER) is the scientific challenge around which gather different communities (signal processing, image processing, artifcial intelligence, robotics, human-computer interaction )

Mehrabian [6] affirms that facial expression represents 55\% of the nonverbal communication that allows to understand the state or the emotion of a person. The objective of this work is to get a computer to detect human emotions from facial expressions.

Facial expression is the most important key to understand human emotions. In fact, not all facial expressions have a meaning and can be classified into emotions, but there are some basic emotions that are universal [7] and can be expressed in the same way, which are: happy, sad, fear, anger, disgust, and surprise.

The main principles steps of facial expression recognition are generally: face detection, feature extraction, and facial expression classification. In the first step; we have to determine whether an image belongs to the class of faces or not. In the second step; we have to extract features or characteristics from the face that better describe emotions. In the last step; we have to classify the extracted features into basic emotions. However, usually the issue comes from the second step which is feature extraction. A set of features that better describes a facial expression movement must be found and used for classification. For this reason, the proposed technique in this paper is based on image sequences, and focused on calculating 1176 euclidean distances between all detected points to measure all possible deformations of the face, because, there may be distances more descriptive than others that appear visually 


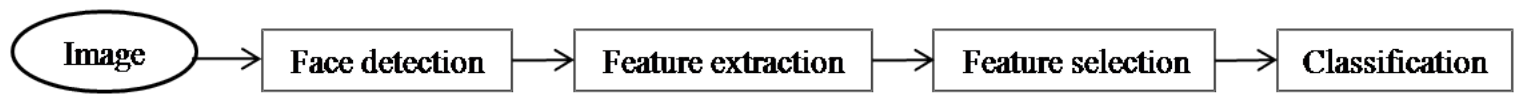

Figure 1. Steps of an emotion recognition system

logic. Once we have calculated dynamic features from fiducial points; an additional step of feature selection process (see Figure 1) is used to reduce the number of features by choosing only the most relevant ones from them.

The rest of the paper is organized as follows: an overview of related work is presented in section 2, our proposed method is presented in section 3, experimental setup is given in section 4, in section 5; results and a discussion of our proposed approach are presented with a comparison of recognition rate between previous works. Section 6 concludes the paper and presents some perspective researches.

\section{RELATED WORK}

In general, facial expression recognition system can be classified into two categories: geometric features based methods and global features based methods [8]. In geometric features-based methods, only some parts of the face are considered for feature extraction such as eyes, nose and mouth. Such methods consume a lot of computation time to obtain accurate results for facial features detection and tracking which is a major disadvantage. Besides, in global features based methods, the whole face is considered for feature extraction, as used in [9] where global features are extracted from face images using local Zernike moment. These methods are easy to use because they work directly on facial images to describe facial textures.

There are a plethora of works that aim to facilitate the way of recognizing emotions from facial expression using static $[10,11,12]$ or dynamic images $[13,14,15,16,17,18,9]$.

By measuring dynamic facial motions in image sequences; Bassili [19] has confirmed that dynamic images give accurate results in facial expression recognition than single static ones.

Friesen et al. [14] have proposed the FACS system that describes movements of the face, where forty four Action Units (AU) are defined, and each one represents a movement of a particular part of the face (e.g Brow Lowerer). According to Friesen et al., a facial expression could be characterized by a combination of AUs.

To demonstrate that AUs are capable to perform emotion expressions, basori et al. [20] have generated an emotion expression of an avatar using combination of AUs based on facial muscle.

Pantic et al. [15] have focused their work on recognizing facial action units (AUs) and their temporal models using profile-view face image sequences. To track 15 facial points in an input face profile sequences; they apply particle filtering method [21].

Valstar et al. [22] have proposed an automatic method to recognize 22 actions units (AUs) and their models using image sequences. Firstly, to automatically detect 20 fiducial points, they used Gabor-feature-based boosted classifier, then, these points were tracked through a sequence of images using a particle filtering method with factorized likelihoods.

$\mathrm{Pu}$ et al. [16] have suggested a new framework for facial expression analysis based on recognizing AUs from image sequences. To detect and track fiducial points, they applied first AAM [23] to model the neutral facial expression in the first frame, after that they used pyramidal implementation of Lucas-Kanade [24] to track feature points in the others frames. They used two levels to classify facial expressions using random forest as method of classification. The first level consists of classifying AUs, taking as input the displacement vectors between the neutral expression frame and the peak expression frame. The second level consists of using as input the detected AUs to classify facial expressions.

Most of facial expression recognition methods are based on AU-based method [15, 22, 16, 20]. They are often influenced by the FACS system proposed by Friesen et al.[14]. Nevertheless, there are also other techniques that are based only on fidicual points to recognize facial expression, which minimize computation time.

Abdat et al. [17] have focused on another geometric method to detect facial expression. They have used twenty one distances to encode facial expressions; these distances describe facial features deformations compared to the neutral state. These methods are focused firstly on the algorithm of Shi\&Thomasi to extract feature points, and secondly on the Lucas-Kanade algorithm [24] to track and detect points, after that the distance vector was used as a descriptor of the facial expression, which is calculated from image sequences. This vector is the input of SVM classifier.

Hammal et al. [13] have developed a classifying system based on the belief theory, and applied it on the Hammal-Caplier database. They used five distances between different parts of the face (eybrow, both eyes and mouth). In their work, distances were computed on skeletons of expression from image sequences, however, only 
four emotions (joy, surprise, disgust and neutral) were considered from the six basic emotions.

Perveen et al. [10] have focused their work on three regions (eyebrows, eyes, mouth) to define an emotion from static images. First, they calculated the characteristic points of the face, then they tried to evaluate some animation parameters such as: the openness of eyes, the width of eyes, the height of eyebrows, the opening of mouth, and the width of mouth. As a classification technique, they used a decision tree based method, applied only on thirty images from the JAFFE database [25], and they recognized six emotions (happy, surprise, fear, sad, angry, and neutral) excluding the disgust emotion.

Saeed et al. [26] have proposed an emotion recognition system based on just eight fiducial points. They represented six geometric features by measuring some distances between mouth, eyes, and eyebrows. These features represent the changes of the face during an emotion occurrence. Then, the features were presented to an SVM classifier for emotion recognition. The system was applied on Cohn-Kanade database (CK+) [27], and Binghamton University 3D Facial Expression Database [28] to recognize six basic emotions.

Majumder et al. [29] have suggested an emotion recognition model based on the Kohonen self-organizing map (KSOM) that uses 26 dimensional facial geometric feature vector calculated from three parts of the face (lips, eyes and eyebrows) that describes the change of six basic emotions. The experience was applied on the MMI database [30].

The research studies cited above show that dynamic facial expressions from image sequences are more descriptive for the task of emotion recognition and can increase the accuracy in real time applications instead of using static images.

\section{PROPOSED METHOD}

This section presents and justifies our proposed technique for emotion recognition from facial expression. Our contribution concerns the feature extraction step, in which we have proposed to calculate all euclidean distances between fiducial points, in the first and in the last frames to measure facial motion. Firstly, we detect the face using ViolaJones algorithm [31], then, we detect and track 49 fiducial points using a powerful and recent Supervised Decent Method (SDM) proposed by Xiong et al. [32], and from these points that represent the four parts of the face (eyebrows, eyes, nose, and mouth), we calculate all possible distances between each pair of points, as a result, we get $C_{49}^{2}=1176$ euclidean distances. After that, to measure dynamic deformation related to the neutral state, we calculate the distance ratio that represents dynamic features, it is calculated between the first and the last frames (Section 2.1). Afterward, we use a feature selection method to reduce the number of features and to select only the most relevant ones. Finally, we present the selected dynamic features to a neural network classifier for facial expression recognition.

\subsection{Facial expression representation}

Once we have detected the face using Viola Jones algorithm [31], we have applied SDM method [32] to detect and track fiducial points in image sequences.

To measure the face deformation, we have considered only the first and the last frames. Firstly, we have calculated all Euclidean distances (1) from 49 detected points that are represented by $\mathrm{x}$ and y coordinates (2) (3), and that refer to the parts of the face which are: 10 points for eyebrows, 12 points for eyes, 9 points for nose, and 18 points for mouth. In the total, we have calculated 1176 distances in the first and in the last frames. Then, we have measured dynamic deformation by calculating the ratio (4) between frames. The ratio represents the division of the calculated distance of the peak frame by the same calculated distance of the first frame. The dynamic features (5) represent a vector of features that contains 1176 ratios calculated related to the neutral state.An overview of facial expression representation process is presented in Figure 2.

$$
\begin{gathered}
D=\left[D_{1}, D_{2}, \ldots, D_{i}, \ldots, D_{t}\right] \\
V_{0}=\left[x_{10}, y_{10}, x_{20}, y_{20}, \ldots, x_{n 0}, y_{n 0}\right] \\
V_{p}=\left[x_{1 p}, y_{1 p}, x_{2 p}, y_{2 p}, \ldots, x_{n p}, y_{n p}\right] \\
\Delta D_{i}=\frac{D_{i p}}{D_{i 0}} \\
D F=\left[\Delta D_{1}, \Delta D_{2}, \ldots, \Delta D_{t}\right]
\end{gathered}
$$

Where

$\mathrm{n}$ : The total number of fiducial points 
$\mathrm{t}$ : The number of euclidean distances calculated between each pair of points

$V_{0}: \mathrm{x}$ and $\mathrm{y}$ coordinates of detected points in the first frame

$V_{p}: \mathrm{x}$ and y coordinates of detected points in the peak frame.

$D_{i p}$ : Euclidean distance in the peak frame.

$D_{i 0}$ : Euclidean distance in the first frame.

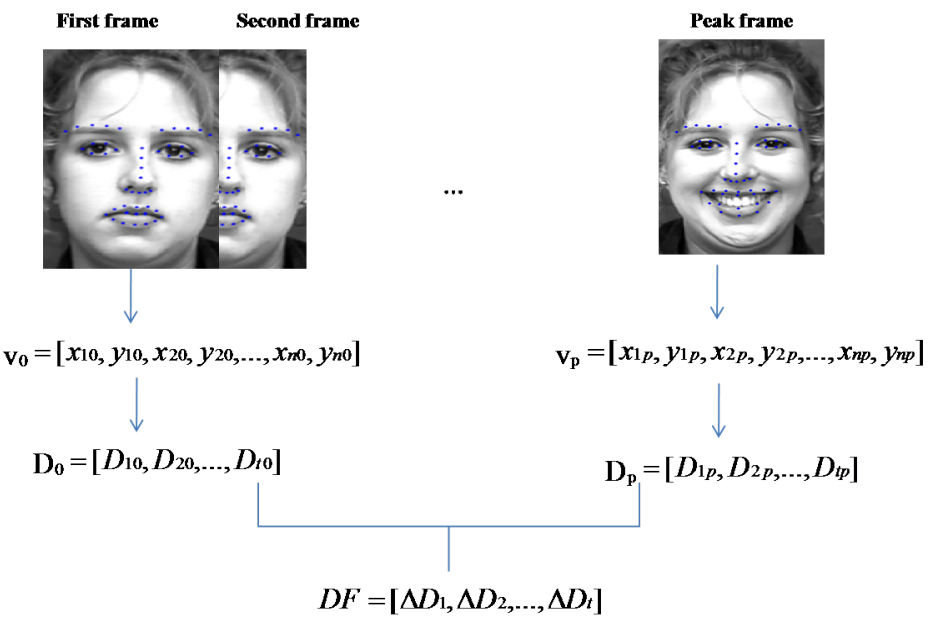

Figure 2. Dynamic features representation process

\subsection{Feature selection}

One of the key issues in emotion classification is the features used for prediction. For this reason; a feature selection step has been used to choose the most relevant features. Generally, the feature selection step combines an attribute subset evaluator with a search method. The attribute evaluator determines what method is used to assign a worth to each subset of features. The search method determines what style of search is performed [33].

In this paper, we have chosen as a feature evaluator the CfsSubsetEval, and Best First as search method implemented in weka. The CfsSubsetEval evaluator evaluates the worth of a subset of features by considering the individual predictive ability of each feature along with the degree of redundancy between them; subsets of features that are highly correlated with the class while having low inter-correlation are preferred. The Best first method searches the space of feature subsets by greedy hill-climbing augmented with a backtracking facility [33].

\subsection{Classification}

A neural network (NN) classifier has been chosen to classify facial expressions based on dynamic features that are previously selected. It was trained on a multi-class emotion recognition task, using the backpropagation algorithmn, and the Sigmoid function as an activation function. Our NN is a signle network with one hidden layer. The first layer represents the input data which are the DF. The second one is the hidden layer, and the last one represents the output classes. The number of neurons in the hidden layer was chosen experimentally.

\section{EXPERIMENTAL SETUP}

The experiments of our work was conducted on three known facial expression databases: Extended CohnKanade (CK+) database [34, 27], Oulu-CASIA VIS database [35] database, and JAFFE database [25].

The CK+ database $[34,27]$ contains 327 labeled image sequences that refer to one of seven expressions, i.e., anger, contempt, disgust, fear, happiness, sadness, and surprise. For each image sequences, only the last frame is provided with an expression label. This database is detailed as follows: 45 images of angry expression, 59 images of disgust expression, 25 images of fear expression, 69 images of happy expression, 28 images of sad expression, and 83 images of surprise expression.

The Oulu-CASIA VIS database [35] contains different light conditions, we have used the strong and good lighting onces that contains 80 subjects. Facial expressions are made by each subject and refer to the six basic expressions (anger, disgust, fear, happiness, sadness, and surprise). In total we have 480 expression labeled image sequences. 
The JAFFE database [25] contains 213 images from 10 Japanese female subjects. Each subject has 3 or 4 examples of each of the six basic expressions (anger, disgust, fear, happiness, sadness, surprise and neutral expression). This database is detailed as follows: 30 images of angry expression, 29 images of disgust expression, 32 images of fear expression, 31 images of happy expression, 31 images of sad expression, 30 images of surprise expression, and 30 images of neutral expression.

\subsection{Training process}

In our work, we have proceeded with three experiments to remark the influence of each used detail on the emotion recognition accuracy. All experiments was conducted on the three databases (CK+, Oulu-CASIA VIS, and JAFFE), and each one has been divided into $60 \%$ for training, $10 \%$ for validation, and $30 \%$ for test, with a NN of 20 neurons in the hidden layer in all experiments.

The first experiment consists firstly; on omitting the feature selection step, and using the DF (5) directly as input to our classifier. Therefore, the NN classifier takes 1176 features as input, and six or seven classes in the output that depend on the number of classes presented in the used database. Secondly; on using the feature selection step. Thus, after calculating DF (5) for each image sequences presented in each used database; we have applied feature selection method on the three databases to reduce the number of features. First, we have combined the $\mathrm{CK}+$, the Oulu-CASIA VIS and the JAFFE databases in one database that contains 1020 data and refers to the eight expressions (anger, contempt, disgust, fear, happiness, sadness, surprise, and neutral). Then, we have applied feature selection method to this new database in order to select the common and only the relevant features. As result, we have reduced our features from 1176 to 83 features. Last, we have trained three classifiers on the three databases, each one apart. The NN classifier takes 83 features as input, and six or seven classes in the output.

In the second experiment, we have tried to observe the ability of classifying new image sequences, the classifier trained on the CK+ was tested on the Oulu-CASIA VIS and the JAFFE databases, and vice versa.

The last experiment consists firstly on unifying the three databases; that means to delete the emotions that don't appear in other databases and keep the common ones. However; it will remain only 309 and 183 image sequences for the CK+ and the JAFFE databases respectively. Secondly, it consists on testing each classifier trained on one database, on the two other databases by varying the size of the training set, and showing how that influences the emotion recognition accuracy.

\section{RESULTS \& DISCUSSION}

Table 1 summarizes a comparaison between the results achieved in our first experiment and those achieved by $\mathrm{Pu}$ et al. [16] using random forest. The third column presents emotion recognition accuracy achieved using directly the DF calculated. The last column presents emotion recognition accuracy using feature selection step where only 83 features are used from 1176 .

The obtained results show that our method outperforms AU based method proposed by [16] whether the feature selection process is used or not. Nevertheless, the use of feature selection process allows to take a less number of features and gives better results than when using DF directly.

Table 1. Comparison of emotion recognition accuracy with and without the use of feature selection process

\begin{tabular}{cccc}
\hline & Pu et al. [16] & \multicolumn{2}{c}{ Our approach } \\
\cline { 3 - 4 } & & Without FS & With FS \\
\hline CK+ & 96.3 & 98 & 99 \\
OULU-CASIA VIS & 76.25 & 81.3 & 84.7 \\
JAFFE & - & 90.6 & 93.8 \\
\hline
\end{tabular}

Table 2 presents the achieved results by the three classifiers trained separately on the $\mathrm{CK}+$, the Oulu-CASIA VIS, and the JAFFE databases, in the second experiment. The first classifier which was trained on the CK+ database using always 83 features, gives an emotion recognition accuracy of $67.29 \%$ and $43.66 \%$ on the Oulu-CASIA VIS, and the JAFFE databases respectively. The second classifier which was trained on the Oulu-CASIA VIS database, gives an emotion recognition accuracy of $90.52 \%$ and $46.48 \%$ on the CK+, and the JAFFE databases respectively. The third classifier which was trained on the JAFFE database, gives an emotion recognition accuracy of $64.22 \%$ and $47.29 \%$ on the CK+, and the Oulu-CASIA VIS databases respectively. We have obtained a competitive emotion recognition accuracy by the second classifier which was trained on the Oulu-CASIA VIS database, unlike classifiers 
Table 2. Test the trained classifier on other databases

\begin{tabular}{ccccc}
\hline & & \multicolumn{3}{c}{ Testing } \\
\cline { 3 - 5 } & & CK+ & OULU-CASIA VIS & JAFFE \\
\hline \multirow{3}{*}{ Training } & CK+ & - & 67.29 & 43.66 \\
& OULU-CASIA VIS & 90.52 & - & 46.48 \\
& JAFFE & 64.22 & 47.29 & - \\
\hline
\end{tabular}

which were trained on the $\mathrm{CK}+$, and the JAFFE databases. However, this decrease of results can be justified firstly by the training size of the CK+ database (196 image sequences) and the training size of the JAFFE database (127 image sequences) comparatively to the size of the Oulu-CASIA VIS database (288 image sequences), secondly, by the expressions which they do not exist on all databases, knowing that the contempt expression is present on the $\mathrm{CK}+$ with an occurrence of 18, but not in other two databases, likewise, in the JAFFE database 30 neutral expressions are considered as emotion class. Therefore, the classifiers trained on a supplementary expressions that do not exist in all databases cause a decreasing of emotion recognition accuracy, for this reason, we have proceeded with the third and last experiment to unify all used databases and show how that influence our results.

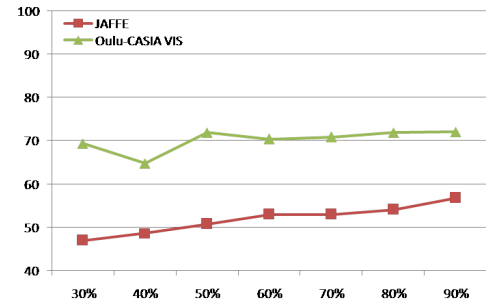

(a) Training on the $\mathrm{CK}+$

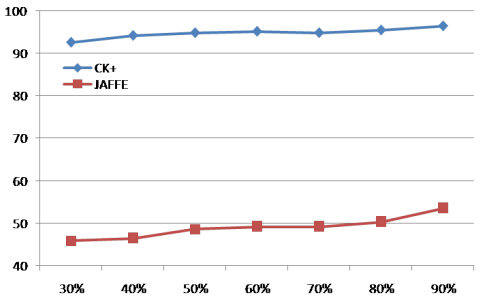

(b) Training on the OULU-CASIA VIS

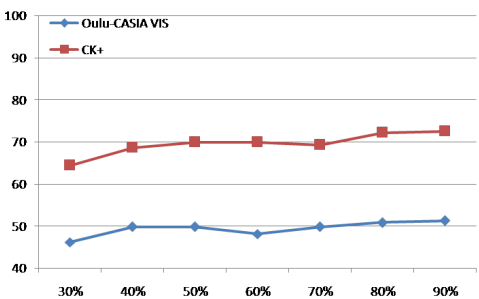

(c) Training on the JAFFE

Figure 3. Training our proposed method with one database and testing it with another databases

Figure 3 shows how the training size and unified databases mark an increase of emotion recognition accuracy over all databases. Figure 3 (a) shows that emotion recognition accuracy increase from $67.29 \%$ to $72.08 \%$ and from $43.66 \%$ to $56.83 \%$ in the Oulu-CASIA VIS and the JAFFE databases respectively. Figure 3 (b) shows that emotion recognition accuracy increase from $90.52 \%$ to $96.44 \%$ and from $46.48 \%$ to $53.55 \%$ in the CK+ and the JAFFE databases respectively. Figure 3 (c) shows that emotion recognition accuracy increase from $64.22 \%$ to $72.49 \%$ and from $47.29 \%$ to $51.25 \%$ in the CK+ and the Oulu-CASIA VIS databases respectively.

\section{CONCLUSION \& FUTURE WORK}

In this research work, we have proposed an automatic approach for facial expression recognition task. Our approach was tested using dynamic features that are calculated from the first and the last frames which represent respectively the neutral state, and an emotional state. After detecting the face and fiducial points in the first and the last frames; all possible euclidian distances have been calculated between each pair of points. For that, we have calculated 1176 distances, then, to measure the deformation; each calculated distance of the first frame is divided by the same calculated distance of the peak frame. After that, we have used a feature selection process to reduce the number of features by choosing only the most relevant ones from them. In the last step of our proposed approach, we have presented the selected dynamic features to a neural network classifier for facial expression recognition.

Evaluating this approach on three known databases has given encouraging results using neural network classifier, with an emotion recognition accuracy of $99 \%$ on the $\mathrm{CK}+$ database, $84.7 \%$ on the Oulu-CASIA VIS database, and $93.8 \%$ on the JAFFE database.

In our future work we will continue developing our proposed system along several axes. Firstly, we will investigate the possibility of adding other features that represent the pose of the face. Secondly, we also intend to consider another source to recognize emotions, which is the intonation of voice, using acoustic parameters. Finally, our ultimate aim is to combine the two sources, which are facial expression and voice intonation, to automatically recognize emotions from multimodal data using new approaches of deep learning classification. 


\section{REFERENCES}

[1] S. Lee and S.-Y. Shin, "Face song player according to facial expressions," International Journal of Electrical and Computer Engineering (IJECE), vol. 6, no. 6, pp. 2805-2809, 2016.

[2] P. Barros, G. I. Parisi, C. Weber, and S. Wermter, "Emotion-modulated attention improves expression recognition: A deep learning model," Neurocomputing, 2017.

[3] J. Arunnehru and M. K. Geetha, "Automatic human emotion recognition in surveillance video," in Intelligent Techniques in Signal Processing for Multimedia Security. Springer, 2017, pp. 321-342.

[4] H. K. Palo and M. N. Mohanty, "Classification of emotional speech of children using probabilistic neural network," International Journal of Electrical and Computer Engineering, vol. 5, no. 2, p. 311, 2015.

[5] S. Motamed, S. Setayeshi, and A. Rabiee, "Speech emotion recognition based on a modified brain emotional learning model," Biologically Inspired Cognitive Architectures, 2017.

[6] A. Mehrabian, "Communication without words," Communication Theory,, pp. 193-200, 2008.

[7] P. Ekman, "An argument for basic emotions," Cognition \& emotion, vol. 6, no. 3-4, pp. 169-200, 1992.

[8] C. Shan, S. Gong, and P. W. McOwan, "Facial expression recognition based on local binary patterns: A comprehensive study," Image and Vision Computing, vol. 27, no. 6, pp. 803-816, 2009.

[9] X. Fan and T. Tjahjadi, "A dynamic framework based on local zernike moment and motion history image for facial expression recognition," Pattern Recognition, vol. 64, pp. 399-406, 2017.

[10] N. Perveen, S. Gupta, and K. Verma, "Facial expression recognition using facial characteristic points and gini index," in Engineering and Systems (SCES), 2012 Students Conference on. IEEE, 2012, pp. 1-6.

[11] F. Z. Salmam, A. Madani, and M. Kissi, "Facial expression recognition using decision trees," in 2016 13th International Conference on Computer Graphics, Imaging and Visualization (CGiV). IEEE, 2016, pp. 125130.

[12] A. T. Lopes, E. de Aguiar, A. F. De Souza, and T. Oliveira-Santos, "Facial expression recognition with convolutional neural networks: Coping with few data and the training sample order," Pattern Recognition, vol. 61, pp. 610-628, 2017.

[13] Z. Hammal, L. Couvreur, A. Caplier, and M. Rombaut, "Facial expression recognition based on the belief theory: comparison with different classifiers," in Image Analysis and Processing-ICIAP 2005. Springer, 2005, pp. 743-752.

[14] E. Friesen and P. Ekman, "Facial action coding system: a technique for the measurement of facial movement," Palo Alto, 1978.

[15] M. Pantic and I. Patras, "Dynamics of facial expression: recognition of facial actions and their temporal segments from face profile image sequences," IEEE Transactions on Systems, Man, and Cybernetics, Part $B$ (Cybernetics), vol. 36, no. 2, pp. 433-449, 2006.

[16] X. Pu, K. Fan, X. Chen, L. Ji, and Z. Zhou, "Facial expression recognition from image sequences using twofold random forest classifier," Neurocomputing, vol. 168, pp. 1173-1180, 2015.

[17] F. Abdat, C. Maaoui, and A. Pruski, "Human-computer interaction using emotion recognition from facial expression," in Computer Modeling and Simulation (EMS), 2011 Fifth UKSim European Symposium on. IEEE, 2011, pp. 196-201.

[18] A. Sánchez, J. V. Ruiz, A. B. Moreno, A. S. Montemayor, J. Hernández, and J. J. Pantrigo, "Differential optical flow applied to automatic facial expression recognition," Neurocomputing, vol. 74, no. 8, pp. 1272-1282, 2011.

[19] J. N. Bassili, "Emotion recognition: the role of facial movement and the relative importance of upper and lower areas of the face." Journal of personality and social psychology, vol. 37, no. 11, p. 2049, 1979.

[20] A. H. Basori and H. M. A. AlJahdali, "Emotional facial expression based on action units and facial muscle," International Journal of Electrical and Computer Engineering (IJECE), vol. 6, no. 5, pp. 2478-2487, 2016.

[21] N. Shepard and M. PITT, "Filtering via simulation: auxiliary particle filter," Journal of the American Statistical Association, vol. 94, pp. 590-599, 1999.

[22] M. F. Valstar and M. Pantic, "Fully automatic recognition of the temporal phases of facial actions," IEEE Transactions on Systems, Man, and Cybernetics, Part B (Cybernetics), vol. 42, no. 1, pp. 28-43, 2012.

[23] I. Matthews and S. Baker, "Active appearance models revisited," International Journal of Computer Vision, vol. 60, no. 2, pp. 135-164, 2004.

[24] J.-Y. Bouguet, "Pyramidal implementation of the affine lucas kanade feature tracker description of the algorithm," Intel Corporation, vol. 5, no. 1-10, p. 4, 2001.

[25] M. J. Lyons, S. Akamatsu, M. Kamachi, J. Gyoba, and J. Budynek, "The japanese female facial expression (jaffe) database," 1998.

[26] A. Saeed, A. Al-Hamadi, R. Niese, and M. Elzobi, "Effective geometric features for human emotion recognition," in Signal Processing (ICSP), 2012 IEEE 11th International Conference on, vol. 1. IEEE, 2012, pp. 
623-627.

[27] P. Lucey, J. F. Cohn, T. Kanade, J. Saragih, Z. Ambadar, and I. Matthews, "The extended cohn-kanade dataset (ck+): A complete dataset for action unit and emotion-specified expression," in Computer Vision and Pattern Recognition Workshops (CVPRW), 2010 IEEE Computer Society Conference on. IEEE, 2010, pp. 94-101.

[28] L. Yin, X. Wei, Y. Sun, J. Wang, and M. J. Rosato, "A 3d facial expression database for facial behavior research," in 7th international conference on automatic face and gesture recognition (FGR06). IEEE, 2006, pp. 211-216.

[29] A. Majumder, L. Behera, and V. K. Subramanian, "Emotion recognition from geometric facial features using self-organizing map,” Pattern Recognition, vol. 47, no. 3, pp. 1282-1293, 2014.

[30] M. Valstar and M. Pantic, "Induced disgust, happiness and surprise: an addition to the mmi facial expression database," in Proc. 3rd Intern. Workshop on EMOTION (satellite of LREC): Corpora for Research on Emotion and Affect, 2010, p. 65.

[31] P. Viola and M. Jones, "Rapid object detection using a boosted cascade of simple features," in Computer Vision and Pattern Recognition, 2001. CVPR 2001. Proceedings of the 2001 IEEE Computer Society Conference on, vol. 1. IEEE, 2001, pp. I-511.

[32] X. Xiong and F. Torre, "Supervised descent method and its applications to face alignment," in Proceedings of the IEEE conference on computer vision and pattern recognition, 2013, pp. 532-539.

[33] M. I. Devi, R. Rajaram, and K. Selvakuberan, "Generating best features for web page classification,” Webology, vol. 5, no. 1, p. 52, 2008.

[34] T. Kanade, J. F. Cohn, and Y. Tian, "Comprehensive database for facial expression analysis," in Automatic Face and Gesture Recognition, 2000. Proceedings. Fourth IEEE International Conference on. IEEE, 2000, pp. 46-53.

[35] G. Zhao, X. Huang, M. Taini, S. Z. Li, and M. Pietikäinen, "Facial expression recognition from near-infrared videos," Image and Vision Computing, vol. 29, no. 9, pp. 607-619, 2011.

\section{BIOGRAPHIES OF AUTHORS}

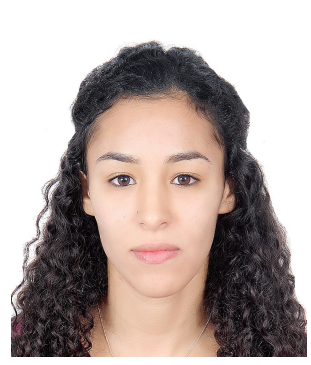

Fatima Zahra SALMAM is a Ph.D student at LAROSERI Laboratory, Faculty of Sciences, University of Chouaib Doukkali, EL Jadida (Morocco). She obtained Master Degree in computer science specialty of Business Intelligence from the University of Sultan Moulay Slimane-Morocco in 2014. Her researches are in fields of emotion recognition, data mining, data analysis, computer vision, and intelligence artificielle. She prepare a dissertation on emotion recognition from image and speech data using data mining techniques.

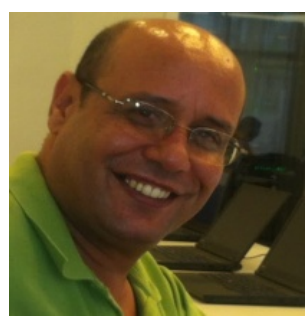

Abdellah Madani is currently a Professor and PhD Tutor in Department of Computer Science, Chouaib Doukkali University, Faculty of Sciences, El Jadida, Morocco. His main research interests include optimization algorithms, text mining, traffic flow and modelling platforms. He is the author of many research papers published at conference proceedings and international journals.



Mohamed Kissi received his PhD degree from the UPMC, France in 2004 in Computer Science. $\mathrm{He}$ is currently a Professor in Department of Computer Science, University Hassan II Casablanca, Faculty of Sciences and Technologies, Mohammedia, Morocco. His current research interests include machine learning, data and text mining (Arabic) and Big Data. He is the author of many research papers published at conference proceedings and international journals in Arabic text mining, bioinformatics, genetic algorithms and fuzzy sets and systems. 